Editorial

\title{
Special issue: Advances in learning schemes for function approximation
}

The eleven papers included in this special issue represent a selection of extended contributions presented at the 11th International Conference on Intelligent Systems Design and Applications (ISDA) held in Córdoba, Spain November 22-24, 2011. Papers were selected on the basis of fundamental ideas and concepts rather than the direct usage of well-established techniques. This special issue is then aimed at practitioners, researchers and postgraduate students, who are engaged in developing and applying, advanced Intelligent Systems to solving real-world problems in the Industrial and Environmental fields. The papers are organized as follows.

In the first contribution, Barros et al., propose a novel BottomUp Oblique Decision-Tree Induction Framework called BUTIF. BUTIF does not rely on an impurity-measure for dividing nodes, since the data resulting from each split is known a priori. BUTIF allows the adoption of distinct clustering algorithms and binary classifiers, respectively, for generating the initial leaves of the tree and the splitting hyperplanes in its internal nodes. It is also capable of performing embedded feature selection, which may reduce the number of features in each hyperplane, thus improving model comprehension. Different from virtually every top-down decision-tree induction algorithm, BUTIF does not require the further execution of a pruning procedure in order to avoid overfitting, due to its bottom-up nature that does not overgrow the tree. Empirical results show the effectiveness of the proposed framework.

The second contribution by Bolón-Canedo et al., propose an ensemble of filters for classification, aimed at achieving a good classification performance together with a reduction in the input dimensionality. This approach overcomes the problem of selecting an appropriate method for each problem at hand, as it is overly dependent on the characteristics of the datasets. The adequacy of using an ensemble of filters rather than a single filter was demonstrated on synthetic and real data, paving the way for its final application over a challenging scenario such as DNA microarray classification.

Cruz-Ramírez et al., in the sequel present a study of the use of a multi-objective optimization approach in the context of ordinal classification and propose a new performance metric, the Maximum Mean Absolute Error (MMAE). MMAE considers per-class distribution of patterns and the magnitude of the errors, both issues being crucial for ordinal regression problems. In addition the authors empirically show that some of the performance metrics are competitive objectives, which justifies the use of multi-objective optimization strategies. In this study, a multi-objective evolutionary algorithm optimizes a artificial neural network ordinal model with different pairs of metrics combinations, concluding that the pair of the Mean Absolute Error (MAE) and the proposed MMAE is the most favorable. A study of the relationship between the metrics of this proposal is performed, and the graphical representation in the 2 dimensional space where the search of the evolutionary algorithm takes place is analyzed. The results obtained show a good classification performance, opening new lines of research in the evaluation and model selection of ordinal classifiers.

In the fourth contribution, Cateni et al., present a novel resampling method for binary classification problems on imbalanced datasets combining an oversampling and an undersampling technique. Several tests have been developed aiming at assessing the efficiency of the proposed method. Four classifiers based, respectively, on Support Vector Machine, Decision Tree, labeled Self Organizing Map and Bayesian Classifiers have been developed and applied for binary classification on the following four datasets: a synthetic dataset, a widely used public dataset and two datasets coming from industrial applications.

In the sequel, Ibañez et al., propose two greedy wrapper forward cost-sensitive selective naive Bayes approaches. Both approaches readjust the probability thresholds of each class to select the class with the minimum expected cost. The first algorithm (CS-SNBAccuracy) considers adding each variable to the model and measures the performance of the resulting model on the training data. In contrast, the second algorithm (CS-SNB-Cost) considers adding variables that reduce the misclassification cost, that is, the distance between the readjusted class and actual class. The authors tested the algorithms on the bibliometric indices prediction area. Considering the popularity of the well-known h-index, they have researched and built several prediction models to forecast the annual increase of the h-index for Neurosciences journals in a four-year time horizon. Results show that the approaches, particularly CS-SNB-Accuracy, often achieved higher accuracy values than other Bayesian classifiers. Furthermore, it has been also noted that the CS-SNB-Cost almost always achieved a lower average cost than the analyzed standard classifiers. These cost-sensitive selective naive Bayes approaches outperform the selective naive Bayes in terms of accuracy and average cost, so the cost-sensitive learning approach could be also applied in different probabilistic classification approaches.

Sobrino et al., in the sixth paper approach causal questions with the aim of: (1) answering what-questions as identifying the cause of an effect; (2) answering how-questions as selecting an appropriate part of a mechanism that relates pairs of cause effect (3) answering why-questions as identifying central causes in the mechanism which answer how-questions. To automatically get answers to why-questions, the authors hypothesize that the deepest knowledge associated 
to them can be obtained from the central nodes of the graph that schematizes the mechanism. This contribution is concerned with medical question answering systems, even though that this approach does not address how to retrieve medical documents as a primary answer to a question, but how to extract relevant causal answers from a given document previously extracted by using a search engine. Thus, this research deals with the automatic detection and extraction of causal relations from medical documents.

In the seventh paper, Sleiman and Corchuelo propose a hybrid approach that explores the use of standard machine-learning techniques to extract web information. The results illustrate that the proposal outperforms three state-of-the-art techniques in the literature, which opens up quite a new approach for information extraction.

García-Hernández et al. in the eighth paper, present a hybrid system for incorporating human expert knowledge into the unequal area facility layout problem. A subset of facility designs is generated using a genetic algorithm and then, evaluated by a human expert. The hybrid system consists of assigning a mark, where the principal aim is to substitute the human expert's knowledge to, avoid fatiguing or burdening him or her. The novel proposed approach was tested using a real case study of 365 facility layout designs for an ovine slaughterhouse. The validation phase of the intelligent model presented was performed using a new subset of 181 facility layout designs evaluated by a different human expert. The results of the experiment, which validate the proposed approach, are presented and discussed in this study.

Kang et al. in the sequel present an effective control method based on adaptive PID neural network and particle swarm optimization (PSO) algorithm. PSO algorithm is introduced to initialize the neural network for improving the convergent speed and preventing weights trapping into local optima. To adapt the initially uncertain and varying parameters in the control system, the authors introduce an improved gradient descent method to adjust the network parameters. The stability of our controller is analyzed according to the Lyapunov method. The simulation of complex nonlinear multiple-input and multiple-output (MIMO) system is presented with strong coupling. Empirical results illustrate that the proposed controller can obtain good precisión with shorter time compared with the other considered methods.

In the tenth paper, Castellano et al. introduce a multi-agent system that exploits positioning information coming from mobile devices to detect the occurrence of user's situations related to social events. In the functional view of the system, the first level of information processing is managed by marking agents which leave marks in the environment in correspondence to the users' positions. The accumulation of marks enables a stigmergic cooperation mechanism, generating short-term memory structures in the local environment. Information provided by such structures is granulated by event agents which associate a certainty degree with each event. Finally, an inference level, managed by situation agents, deduces user situations from the underlying events by exploiting fuzzy rules whose parameters are generated automatically by a neuro-fuzzy approach. Fuzziness allows the system to cope with the uncertainty of the events. In the architectural view of the system, the authors adopt semantic web standards to guarantee structural interoperability in an open application environment. The system has been tested on different real-world scenarios to show the effectiveness of the proposed approach.

Chira et al. in the final paper modeled the real-world optimization problem of urban bicycles renting systems as a capacitated Vehicle Routing Problem (VRP) with multiple depots and the simultaneous need for pickup and delivery at each base station location. Evolutionary algorithms and ant colony systems are proposed and real data from the cities of Barcelona and Valencia is used for experimental simulations.

We would like to thank our peer-reviewers for their diligent work and efficient efforts. We are also grateful to the Editor-in-Chief of Neuro-computing, Prof. Tom Heskes, for his continued support for the ISDA conference and for the opportunity to organize this Special issue.

\section{Acknowledgments}

This research is partially supported by the Spanish Ministry of Economy and Competitiveness under Project TIN2010-21272-C02-01 (funded by the European Region-al Development Fund) and SA405A12-2 from Junta de Castilla y León. This work was also supported in the framework of the IT4 Innovations Centre of Excellence Project, Reg. no. CZ.1.05/1.1.00/02.0070 by operational program 'Research and Development for Innovations' funded by the Structural Funds of the European Union and state budget of the Czech Republic, EU. The research work of P.A. Gutiérrez is funded by the TIN2011-22794 project of the Spanish Ministerial Commission of Science and Technology (MICYT), FEDER funds and the P11-TIC-7508 project of the "Junta de Andalucía" (Spain).

\section{Guest Editors}

Emilio Corchado*

Departamento de Informática y Automática, Universidad de Salamanca, Plaza de la Merced S/N, 37008 Salamanca, Spain E-mail address: escorchado@usal.es

Ajith Abraham Machine Intelligence Research Labs (MIR Labs), Scientific Network for Innovation and Research Excellence, P.O. Box 2259, Auburn, WA 98071, USA

IT4Innovations, European Center for Excelence, VSB-Technical University of Ostrava, 17. listopadu 15, 70833 Ostrava, Poruba, Czech Republic

E-mail address: ajith.abraham@ieee.org

Pedro Antonio Gutiérrez Departamento de Informática y Análisis Numérico, Universidad de Córdoba, Campus Rabanales, Anexo del Edificio Marie Curie (antiguo C3), $1^{a}$ Planta, 14071 Córdoba, Spain E-mail address: pagutierrez@uco.es

José Manuel Benítez Departamento de Ciencias de la Computación e Inteligencia Artificial, Universidad de Granada, C/ Daniel Saucedo Aranda, s/n D31 (4 $4^{a}$ planta), 18071 Granada, Spain E-mail address: j.m.benitez@decsai.ugr.es

Sebastián Ventura Departamento de Informática y Análisis Numérico, Universidad de Córdoba, Campus Universitario de Rabanales, Edificio “Albert Einstein”, 3ª Planta, 14071 Córdoba, Spain E-mail address: sventura@uco.es

Received 8 December 2013; accepted 15 December 2013 Available online 2 January 2014 